\title{
A Case of Abducense Nerve Palsy as a Rare Feature of Tuberculosis Meningoencephalitis
}

\author{
Mohammad Rahmanian ${ }^{1}$, Zahra Mosallanezhad ${ }^{2}$, Mohamed Amin Ghobadifar ${ }^{3}$ and Safar Zarei*4 \\ ${ }^{1}$ Department of Anesthesiology, Shiraz University of Medical Sciences, Iran
}

${ }^{2}$ Department of Obstetrics and Gynecology, Jahrom University of Medical Sciences, Iran

${ }^{3}$ Zoonoses Research Center, Jahrom University of Medical Sciences, Iran

${ }^{4}$ Department of Physiology, Jahrom University of Medical Sciences, Iran

Received: May 17, 2018; Published: May 23, 2018

*Corresponding author: Safar Zarei, Department of Physiology, Jahrom University of Medical Sciences, Jahrom, Motahari Avenue, postal code 193, Jahrom, Iran

\section{Introduction}

The exact etiology of encephalitis is often unknown and a high rate of morbidity and mortality is associated with it. Number of studies in North America and Europe was done to find the etiology of encephalitis whether infectious or not, during the last decades [1,2]. In this regard, a meta-analysis of a 41 analyzed studies showed that the most etiology of encephalitis is absence [3]. The most severe site of tuberculosis infection is tuberculosis encephalitis with recurrently atypical evolution and onset, an intensely polymorphic disease, and with rarely established etiological diagnosis [1]. In spite of adequate treatment, tuberculosis encephalitis has a severe prognosis. Thus, tuberculosis encephalitis is known to be one of a severe health problem with high mortality rates. On the other hand, lack of safe and fast diagnostic algorithm often causes late specific treatment which finally increases the incidence of tuberculosis in general population [3]. Even though, tuberculosis meningitis is well defined, marked features of encephalitic are reported less commonly. Given this background, we aimed to describe the rare features of a case with tuberculosis encephalitis in this report.

\section{Case Report}

A 17-year-old woman from Iran country was admitted to Peymanieh hospital affiliated with Jahrom University of Medical Sciences complaining of fever, anorexia, photosensitivity, headache, agitation, confusion, impaired health status, positive signs of meningeal irritation, and diplopia of 2 days duration which developed overnight.

The onset of disease with manifestation of fever, anorexia, and photosensitivity was about 15 days before hospitalization. The patient referred to an outpatient clinic and was evaluated by an internist. He received acetaminophen for his fever and headache and discharged. She didn't become well, therefore transferred to our hospital.

On admission: blood pressure 120/80 mmHg, heart rate 89/ min, respiratory and hemodynamic stable state, normal weight, confusion, influences general state, positive signs of meningeal irritation, diffuse headache, double vision of 2 days duration. A neurological examination revealed isolated right abducense nerve palsy which manifested as esotropia in primary gaze and paralysis of lateral rectus muscle on the right, but with no obvious papillary defect. Visual acuity and other cranial nerves were normal during examination. The paraclinical tests showed normal white blood cells count (5630/mm3) neutrophilia (64\%), elevated erythrocyte sedimentation rate $(105 \mathrm{ml} / \mathrm{hr})$, positive C-reactive protein. The spiral CT-scan of the chest without contrast revealed multiple small milliary nodules in both lung fields. Biopsy tissue of lung showed no malignant cells, but polymerase chain reaction for Mycobacterium Tuberculosis was positive. A lumbar puncture was done which revealed pleocytosis (523 elem/mm3), 70\% lymphocytosis, hight proteinorrhachia (112 mg/dl), low glycorrhachia (29 mg/dl).

By suspicious to meningo-encephalitis tuberculosis which is confirmed by positive Lowenstein-Jensen culture for Mycobacterium Tuberculosis, we started treatment against tuberculosis with four agents together including (Rifampicin $750 \mathrm{mg}+$ Ethambutol $1.5 \mathrm{~g}+$ Isoniazide $250 \mathrm{mg}+$ Pyrazinamide $1.5 \mathrm{~g}$ ) with intravenous Ciprofloxacin. To reduce barin edema, dexamethasone was also injected. During treatment, because of increasing transaminase levels, we decreased Rifampicin doses. After 40 days treatment on these medications, her diplopia was disappeared with favorable evolution, and finally discharged in good general state, good orien- 
tation, without fever and headache, and all general and neurological examinations were being normal.

\section{Discussion}

Features of prominent encephalitic are less commonly reported in spite of well description of tuberculous meningitis. The stage of disease at diagnosis may show the death and illness rate of neurotuberculosis. Tuberculous meningitis is associated with significant mortality and morbidity because of difficult in diagnosis which is dependent to a long term of therapy. Changes in mentation, fever, meningismus, headache and focal neurologic deficits are symptoms and signs of neurotuberculosis [2]. Ophthalmic pathologies and its complications such as; homonymous hemianopia, conjunctivitis, choroidal tuberculoma, third or sixth nerve palsies, and nystagmus have also been reported. The oculomotor nerve involvement can happen because of basal meningitis in the base of brain, due to a tuberculoma positioned in the midbrain region [3]. Three different types of categorize for this disease are described based on examination findings.

Headache, malaise, and fever without cranial nerve impairment are described as Stage 1. Paresis and focal seizures due to single cranial nerve impairment are the presence of Stage 2. High rate of neurologic sequelae despite therapy such as multiple cranial nerve paraplegia, palsies, or hemiparesis, and comatose or stuporous have marked as Stage 3. Furthermore, optic nerve and third nerve involvement are associated with stage 3 of tubercular Meningoencephalitis. Neuro-ophthalmic features which are common in the elderly are associated with stage 3 of tubercular Meningoencephalitis [1]. Appropriate therapy with the timing of the initiation may improve the prognosis of tuberculous meningitis. Severe neurological sequelae and poor prognoses are results of delays in treatment and diagnosis [3]. In summary, when a patient with tuberculous meningitis presents with abducense nerve palsy, rapid re-diagnosis should be undertaken and proper treatment initiated, because the prognosis is critically dependent on the timing of adequate treatment.

\section{References}

1. Granerod J, Ambrose HE, Davies NW, Clewley JP, Walsh AL, et al. (2010) Causes of encephalitis and differences in their clinical presentations in England: a multicentre, population-based prospective study. Lancet Infect Dis 10(12): 835-844.

2. Mailles A, Stahl JP (2009) Infectious encephalitis in france in 2007: a national prospective study. Clin Infect Dis 49(12): 1838-1847.

3. Granerod J, Tam CC, Crowcroft NS, Davies NW, Borchert M, et al. (2010) Challenge of the unknown. A systematic review of acute encephalitis in non-outbreak situations. Neurology 75(10): 924-32.

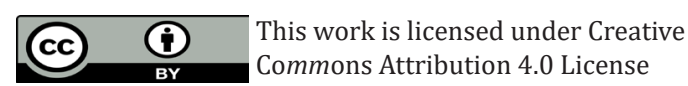

Submission Link: https://biomedres.us/submit-manuscript.php

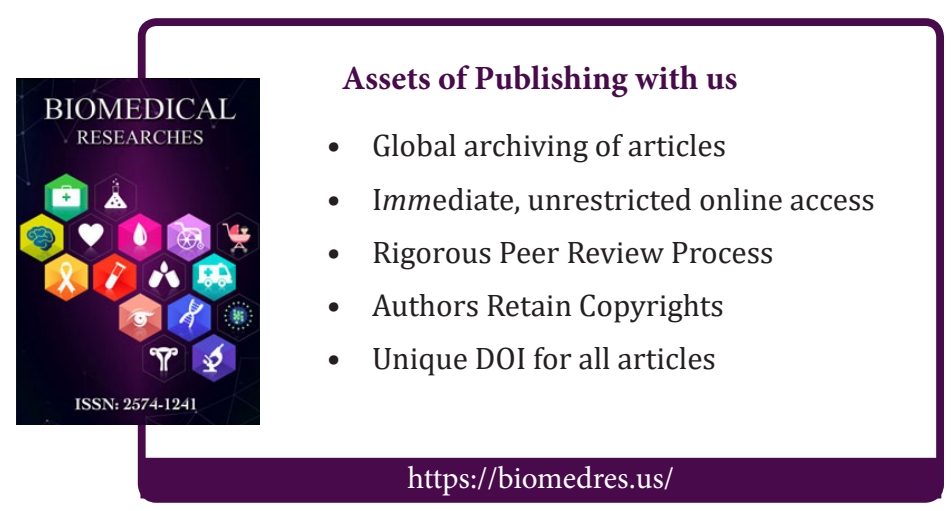

